Aus dem Pathologisch-anatomischen Institut des Stădtischen Krankenhauses in Christiania.

(Vorstand: Prosektor E. H. Hansteen.)

\title{
Ueber die Herzgröße bei verschiedenen Krankheiten.
}

Vorläufige Mitteilung.

Von Sofns Wideröe, Assistenzarzt.

Obgleich in letzter Zeit verschiedene Arbeiten über die patho logischen Veränderungen der HerzgröBe erschienen sind (Hasenfeld Hirsch, Schubert), kann man doch durchaus nicht behaupten, daB diese im Detail vollständig gek]ārt seien. In der Müllerschen Wägungamethode haben wir ein genaues Verfahren erhalten. Nur diese ermöglicht eine exakte Vorstellung über dje relativen Sohwankungen in der Größe der pathologisch veränderten Herzabschnitte. W. Müller hat anf Grund seines großien Materials einzelne Verhälthisse berechnet, z. B. die „Herzproportion" und dio Relation zwisohen den einzelnen Herzkammern. Er hat die Durchschnittsgewichte für die verschiedenen Herzabschnitte für jedes Alter und Geschlecht gefunden. Die Müllersche Methode ist noch relativ wenig benutzt worden, und die Ergebnisse, die man damit bekommen hat, sind teilweise falsch, weil man dje Müllerschen Durchschnittszahlen mit den pathologischen Gewichten verglichen hat. $\mathrm{Fs}$ ist einleuchtend, daß man keine korrekten Resultate erhalten kann, wenn man einzelne pathologische Fälle mit einer Durchschnittazahl vergleicht, und die bei der Müller schen generalisierenden Methode gefundenen Zahlen kann man auf diese Weise nicht benutzen.

Richtiger ist es, die relativen Verhältnisse zu berechnen und die respektiven Proportionen miteinander zu vergleichon, die patholo. gischen mit den physiologischen. Hat mandie normalen Proportionen bestimmt, so ist man berechtigt. die Proportionen jedes pathologischen Falles mit diesen zu vergleichen. Niemand ist bis jetzt auf dieses Moment aufmerksam geworden; die normalen Proportionen sind auch nicht bestimmt.

Ich hobe versucht, die Verände. rungen der Herzgröße nur unter Anwendung relativer Zahlen gu beschreiben, und möchte einen kurzen Ueberblick über meine Resultate hier mitteilen.

Es ist durch frühere Untersuchungen nachgewiesen, daß nur der Herzabschnitt hypertrophiert, auf welchen das pathologische Mo. ment einwirkt, gleichzeitige Hypertrophie anderer Herzabschnitte ist susgesohlossen. Jeder Herzab. schnitt steht also in funktioneller Hinsicht nur in Bezlehungzir den

Momenten, die ihn beeinflussen

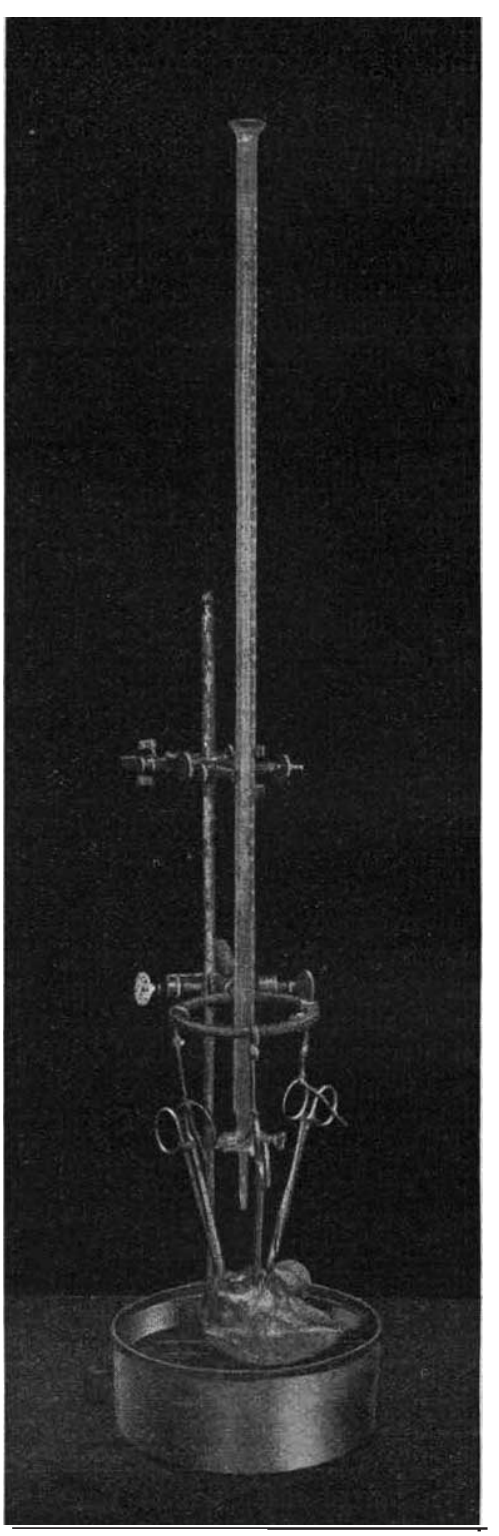
köngen. Da es runmöglich ist, den

Septumteil der Herzkammern unter pathologischen Verhaltnissen zu be. stimmen, habe ich das Septum in die Berechnung nicht aufgenommen.

Von den von mir untersuchten und berechneten Verhältnissen werde ich hier folgende besprechen:

1. Linker Ventrikel: Körpergewicht. Diese Proportion dräckt die absolute Horzgröße aus. Bestimmung nach der Müllerschen Methode. Am größten habe ich diesen Index unter physiolo. gischen Bedingungen bei sehr kleinen Kindern gefunden. Im mittleren Altor ist er niedriger, um im Greisenalter otwas größer zu werden. Durchschnittlich ist dieser Index für beide Geschlechter gleich 1,8, d. h. : 1,8 g Muskulatur des linkenVentrikels trifft auf jedes Kilogramm Körpergewicht.

2. Rechter Ventrikel: Linker Ventrikel. Dieser Index ist ziemlich konstant vom dritten Monat des Lebens an, durehschnittlich gleich 0,57 .

3. Reohter Vorhof: Linker Vorhof $=1,00$. Hat wenig Interesse, 
4. Kapazität des rechten Ventrikels: Kapazität des linken Ventrikels. Bei plötzlich verstorbenen Individuen ist dieser Index gleich 1,06. Um die Kapazität der Ventrikel zu messen, habe ich folgende Methode benutzt: Die Vorhöfe werden an der Atrioventrikulargrenze abgeschnitten. Nachdem die Herzkammern von Blut und Gerinnseln gereinigt sind, wird die Totenstarre beseitigt, was mir durch massageähnliche Manipulationen in einigen Minuten gelang. Mit drei Kocherschen Pinzetten, die sorgfältig an der Atrioventrikulargrenze befestigt sind, wird das Herz in Wasser suspendiert gehalten, wie man es auf der Photographie sieht. Die abführende Arterie ist mit einer großen Arterienpinzette geschlossen. Die Messungen wurden für jede Herzkammer dreimal ausgeführt, die Mittelzahl wurde notiert. Im. ganzen habe ich diese Relationen bei 311 Herzen bestimmt.

Die Resultate sind graphisch dargestellt (s. Kurve).

Kurve 1.

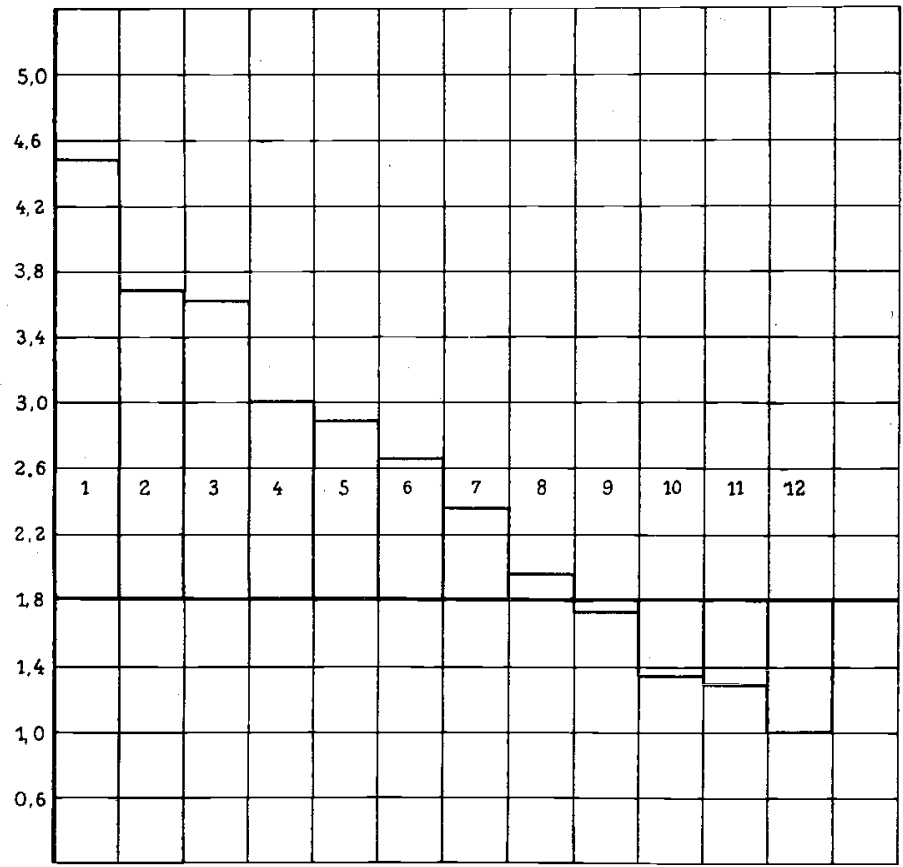

Sie zeigt die Schwankungen der Proportion ,Linker Ventrikel Körpergewicht" bei verschiedenen Krankheiten: 1. Hypernephrom, 2. Pericarditis chronica adhaesiva, 3. Nephritis chronica, 4. Aortenfehler, 5. Arteriosklerose, 6. Insufficientia mitralis, 7. Kyphoskoliose, 8. Tuberculosis pulmonum, 9. Stenosis mitralis, 10. Carcinoma, 11. Chronische, nicht tuberkulöse Lungenkrankheiten, 12. Adipositas excessiva.

Die pathologischen Variationen des Ventrikelindex sieht man auf Kurve 2.

Kurve 2.

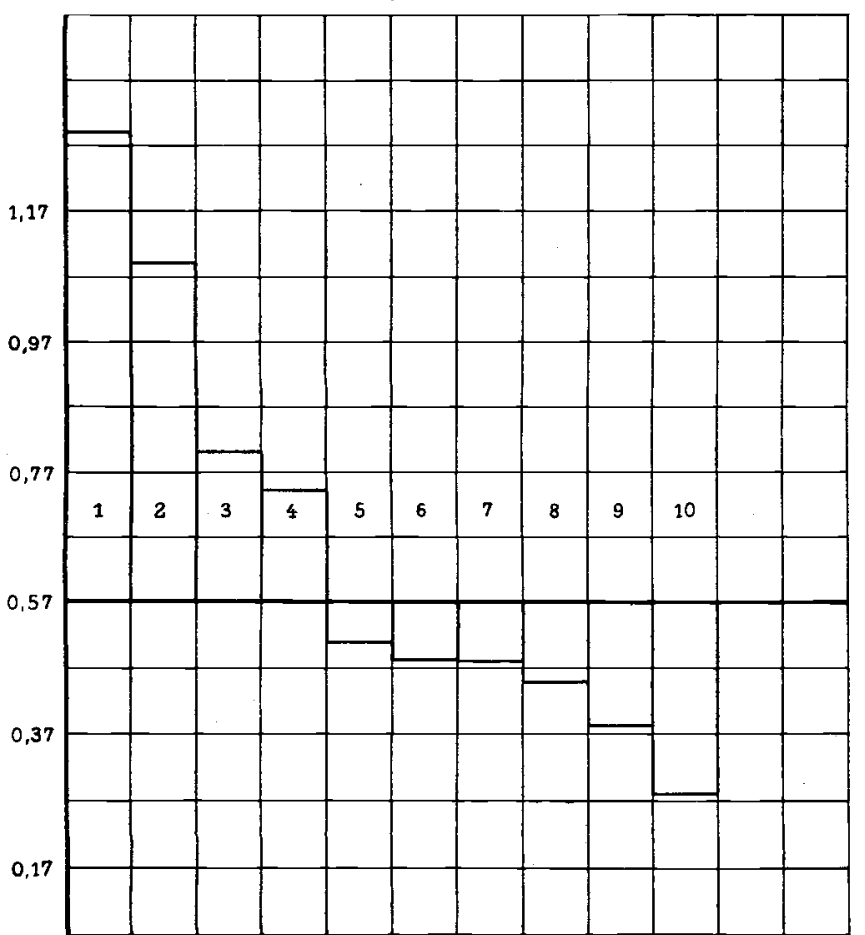

1. Chronische, nicht tuberkulöse Lungenkrankheiten, 2. Stenosis mitralis, 3. Tuberculosis pulmonum, 4. Kyphoskoliose, 5. Pericarditis chronica adhaesiva, 6. Insufficientia mitralis, 7.Aortenfehler, 8. Nephritis chronica, 9. Arteriosklerose, 10. Hypernephrom.
Das Verhältnis zwischen den Kapazitäten der beiden Ventrikel unter pathologischen Bedingungen zeigt Kurve 3 .

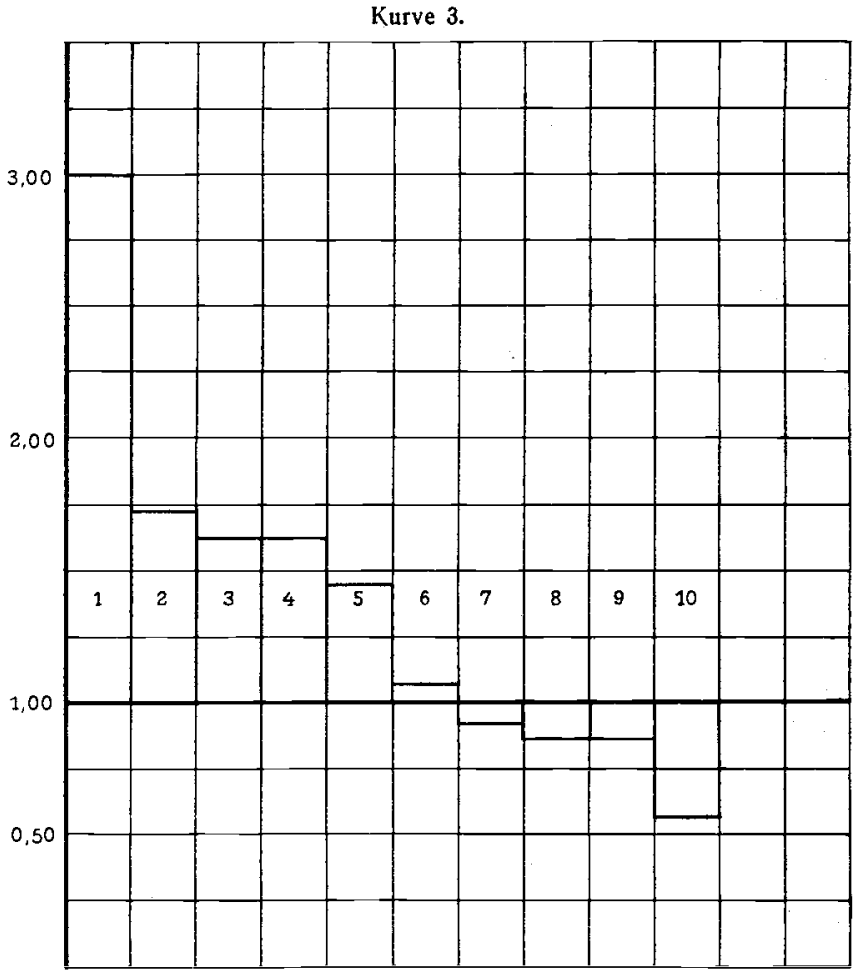

1. Stenosis mitralis, 2. Kyphoskoliosis, 3. Tuberculosis pulmonum, 4. Chronische, nicht tuberkulöse Lungenkrankheiten, 5. Aortenfehler, 6. Insufficientia mitralis, 7. Nephritis chronica, 8. Arteriosklerose, 9. Hypernephrom, 10. Pericarditis chronica adhaesiva.

Meine Resultate weichen in vielen Beziehungen von den gewöhnlichen ab. Zum Beispiel habe ich im Gegensatze zu Hirsch eine bedeutende Hypertrophie bei Obliteration der Perikardialhöhle gefunden. Bei chronischer Nephritis fand ich im Anfangsstadium eine allgemeine Herzhypertrophie, in den terminalen Stadien dagegen eine ausgeprägte linkseitige Hypertrophie. Peripherische Arterio. sklerose ist gewöhnlich mit einer beträchtlichen Herzhyper. trophie verbunden, Arteriosklerose der Aorta dagegen mit einer geringen. Durch experimentelle Hydrämie gelingt es nicht, Herzhypertrophie hervorzurufen. Ueber sonstige Einzelheiten und über die theoretische Begründung meiner Resultate werde ich anderwärts berichten. 